This item was submitted to Loughborough's Research Repository by the author.

Items in Figshare are protected by copyright, with all rights reserved, unless otherwise indicated.

\title{
Scalable synthesis of multicolour conjugated polymer nanoparticles via Suzuki-Miyaura polymerisation in a miniemulsion and application in bioimaging
}

\section{PLEASE CITE THE PUBLISHED VERSION}

http://dx.doi.org/10.1016/j.reactfunctpolym.2016.08.006

\section{PUBLISHER}

(C) Elsevier B.V.

\section{VERSION}

AM (Accepted Manuscript)

\section{PUBLISHER STATEMENT}

This work is made available according to the conditions of the Creative Commons Attribution-NonCommercialNoDerivatives 4.0 International (CC BY-NC-ND 4.0) licence. Full details of this licence are available at: https://creativecommons.org/licenses/by-nc-nd/4.0/

\section{LICENCE}

CC BY-NC-ND 4.0

\section{REPOSITORY RECORD}

Behrendt, Jonathan M., Jair A. Esquivel Guzman, Laura Purdie, Helen Willcock, John J. Morrison, Andrew B. Foster, Rachel K. O'Reilly, Mark C. McCairn, and Michael L. Turner. 2017. "Scalable Synthesis of Multicolour Conjugated Polymer Nanoparticles via Suzuki-miyaura Polymerisation in a Miniemulsion and Application in Bioimaging". figshare. https://hdl.handle.net/2134/24042. 
Scalable Synthesis of Multicolour Conjugated Polymer Nanoparticles via SuzukiMiyaura Polymerisation in a Miniemulsion and Application in Bioimaging

Jonathan M. Behrendt ${ }^{\mathrm{a}, 1}$, Jair A. Esquivel Guzman ${ }^{\mathrm{a}}$, Laura Purdie ${ }^{\mathrm{b}}$, Helen Willcock ${ }^{\mathrm{c}, 2}$, John J. Morrison ${ }^{\mathrm{a}}$, Andrew B. Foster ${ }^{\mathrm{a}}$, Rachel K. O’Reilly ${ }^{\mathrm{c}}$, Mark C. McCairn ${ }^{\mathrm{a}}$, Michael L. Turner $^{\mathrm{a}^{*}}$

a School of Chemistry, University of Manchester, Oxford Road, Manchester, M13 9PL, U.K.

b School of Pharmacy, University of Nottingham, University Park, Nottingham, NG7 2RD, U.K.

c Department of Chemistry, University of Warwick, Gibbet Hill, Coventry, CV4 7AL, U.K.

Corresponding Author

* University of Manchester. Oxford Road, Manchester M13 9PL, UK. Email: michael.turner@manchester.ac.uk Fax:+44(0)1612754273; Tel: +44(0)1612754625.

Present Addresses

1 (J.M.B.) Cambridge Display Technology, Unit 12, Cardinal Park, Cardinal Way, Godmanchester PE29 2XG, United Kingdom.

2 (H.W.) Department of Materials, Loughborough University, Loughborough LE11 3TU, United Kingdom. 


\begin{abstract}
Suzuki cross-coupling polymerisation of aryldibromides and aryldiboronate esters in a sodium dodecyl sulfate (SDS)-stabilised miniemulsion provides a versatile and direct route to fluorescent conjugated polymer nanoparticles (CPNs). These nanoparticles have a conjugated backbone based on poly(9,9-dioctylfluorene) (PFO), however, significant structural diversity is introduced by incorporation of electron withdrawing, heterocyclic comonomers (5-50 mol. $\%)$ in order to tune the emission wavelengths from blue to far-red/near-infrared. The robust nature of the polymerisation methodology allows for rapid assessment of the relationship between polymer composition, chain morphology and optical properties of the resultant CPNs. Moreover, the CPNs (after a simple and rapid purification step) can be used directly in fluorescence-based intracellular labelling experiments (in HCT116 cells), in which they display low cytotoxicity at biologically-useful concentrations.
\end{abstract}

\title{
Keywords
}

conjugated polymer nanoparticles, miniemulsion, Suzuki-Miyaura polymerisation, scalable, bioimaging

\section{Introduction}

Conjugated polymer nanoparticles (CPNs), sometimes referred to as conjugated polymer dots, have been examined for a wide range of fluorescence-based cellular labelling and biomedical imaging applications. In vitro, CPNs display low cytotoxicity and can be internalized by cells through several active mechanisms that depend on the size and nature of the nanoparticle surface.[1-4] Recent developments include the use of CPNs for in vivo targeted bioimaging of tumours, highlighting the potential of these materials as molecular imaging probes in a clinical setting.[5-9] CPNs do not show the undesirable fluorescence 
blinking behaviour and inherent toxicity associated with heavy-metal (e.g. Cd, $\mathrm{Pb}$ ) derived quantum dots that are often used in similar applications.[10, 11] Two methods for the formation of CPNs have dominated literature reports to-date: i) nanoprecipitation, in which a dilute solution of a conjugated polymer (CP) in a water miscible, 'good solvent' (usually tetrahydrofuran) is added rapidly to a non-solvent (water),[12-14] and ii) miniemulsion, in which a solution of a CP in a non-water miscible, volatile solvent (e. g. dichloromethane) is emulsified in an aqueous solution of an appropriate surfactant, followed by solvent evaporation.[15-17] Both of these processing techniques are relatively straightforward and applicable to a range of commercially available CPs. The major limitation of these approaches is a lack of scalability: nanoprecipitation is particularly problematic in this respect in that it can only be achieved using very dilute polymer solutions and thus the resultant CPN dispersions have low solids contents (typically $<500$ ppm). Miniemulsion of pre-formed CPs can be used to produce stable CPN dispersions at higher concentrations, but this approach necessitates the use of a multistep procedure in which the CP is initially prepared and isolated using conventional synthetic methods prior to subsequent dispersion in the miniemulsion.

In an effort to minimize these limitations, various research groups have developed synthetic routes to CPNs in which the polymerisation and nanoparticle formation are achieved concurrently.[7, 18-24] The various classes of light-emitting CPs from which formation of CPNs is desirable are structurally diverse and, moreover, their optical bandgap (and consequently their fluorescence emission wavelength) is directly controlled by the chemical composition of the polyaromatic backbone. Consequently, the scope of any direct synthetic route to CPNs is very much reliant on the versatility of the underlying polymerisation technique. Weder et al. pioneered this one-pot approach to CPNs, through their early report of the synthesis of hyperbranched poly(phenylene ethynylene) (PPE) nanoparticles via Sonogashira coupling of $\mathrm{A}_{2}+\mathrm{B}_{2}+\mathrm{A}_{3}$ monomers (where $\mathrm{A}=$ bromo/iodo, $\mathrm{B}=$ ethynyl) in 
emulsified droplets of toluene in an aqueous solution of the ionic surfactant sodium dodecylsulfate (SDS).[18] Most notably, the authors demonstrated that use of an ultrasonic bath for emulsification enabled the synthesis of fluorescent nanoparticles with average diameters ranging from 400-500 $\mathrm{nm}$. The scope for heterogeneous Sonogashira polymerisation reactions to be used in the direct synthesis of PPE-based CPNs has since been considerably extended by Mecking et al.[19] Here the authors focused on $A_{2}+B_{2}$ stepgrowth reactions, employing the same catalytic system $\left(\mathrm{Pd}\left(\mathrm{PPh}_{3}\right)_{4}, \mathrm{CuI}\right.$, diisopropylamine), dispersed solvent (toluene) and surfactant (SDS) as the earlier report. More rigorous sonication prior to polymerisation was found to enable the formation of smaller nanoparticles (average diameter $=60-120 \mathrm{~nm}$ ), which were of an appropriate size for use in intracellular imaging of HeLa cells. Moreover, it was demonstrated that incorporation of varying amounts of fluorenone or pyrolopyrrole comonomers into the polymer backbone provided access to blue, green or far-red/near infrared (FR/NIR) emitting CPNs.

Suzuki coupling is widely used for the synthesis of both linear and branched CPs. Unlike Sonogashira coupling, Suzuki reactions involve aryl-aryl coupling (of aryl bromides with aryl boronic acids/boronate esters) and the resultant CPs thus have fully aromatic $\pi$-conjugated backbones. Due to the ubiquity of Suzuki coupling in CP synthesis, many appropriate, mutually-reactive $\mathrm{A}_{2}$ and $\mathrm{B}_{2}$ monomers (where $\mathrm{A}=$ bromo, $\mathrm{B}=$ boronic acid/boronate esters) are commercially available. There have been various attempts to develop heterogeneous Suzuki polymerisation reactions for the direct synthesis of CPNs. [21-24] Liu et al. have explored the use of a non-ionic surfactant (Tween 80) for the heterogeneous, Suzuki polymerisation reaction of either 2,7-dibromo-9,9-dioctylfluorene or 4,7-dibromo-2,1,3benzothiadiazole with a slight excess of 9,9-dioctylfluorene-2,7-diboronic acid bis(1,3propanediol) ester and a small amount of 1,3,5-tribromobenzene (2 mol. \%).[21] These reactions provide blue or green fluorescent CPNs (average diameter $=100-200 \mathrm{~nm}$ ) 
comprised of CPs with a hyperbranched structure. We have recently demonstrated that when carried out at room temperature, Suzuki emulsion polymerisation reactions with Triton-X102 as surfactant produce either rod-like or spherical nanoparticles, depending on the chemical structure of the conjugated polymer.[24]

The use of ionic surfactants in heterogeneous Suzuki polymerisation has advantages for emulsion stability where higher temperatures are required and also makes surfactant removal during purification steps less challenging. Ionic surfactants typically have higher critical micelle concentrations (CMCs) and lower micellar molecular weights (MMWs) than nonionic surfactants, rendering them readily amenable to removal by dialysis. Furthermore, ionic surfactants often have much higher cloud points than their non-ionic counterparts (usually > $\left.100{ }^{\circ} \mathrm{C}\right)$. Mecking and co-workers have reported the direct synthesis of poly(9,9dioctylfluorene) CPNs via the Suzuki polymerisation reaction of an A-B type monomer, 2(4,4,5,5-tetramethyl-1,3,2-dioxaborolan-2-yl)-7-bromo-9,9-dioctylfluorene, in an SDSstabilised miniemulsion using $\mathrm{Pd}\left(\mathrm{PPh}_{3}\right)_{4}$ as a catalyst and sodium hydroxide as base.[23]

\section{$<$ Scheme 1 inserted her $>$}

Destabilisation of the miniemulsion was avoided by using the minimum amount of base (2 mol. equivalents) necessary to promote the catalytic reaction. A similar approach has since been employed for the synthesis of CPNs by reaction of 9,9-dioctylfluorene-2,7-diboronic acid bis(1,3-propane-diol) ester and 1,3,5-dibromobenzene in a miniemulsion that was stabilised by the cationic surfactant cetyl trimethylammonium bromide.[22] Both of these reports provide useful strategies for the direct synthesis of CPNs with relatively small diameters (ca. 40-150 nm), but are limited in their scope in that both reports focused on the preparation of fluorene homopolymers. 
In order to demonstrate that Suzuki miniemulsion polymerisation reactions are robust, generally applicable and afford considerable structural diversity, we herein report the use of this technique for the polymerisation of a range of $A_{2}+B_{2}$ type monomers. All reactions contain $50 \mathrm{~mol}$. \% of 2,2'-(9,9-dioctyl-9H-fluorene-2,7-diyl)bis(4,4,5,5-tetramethyl-1,3,2dioxaborolane), 2 , as the $\mathrm{B}_{2}$ monomer: structural variety is achieved through reaction with different $\mathrm{A}_{2}$ comonomers to copolymers of poly(9,9-di-n-octylfluorenyl-2,7-diyl) (PFO). This approach provides access to either alternating co-polymers (with $50 \mathrm{~mol}$. \% of a single $\mathrm{A}_{2}$ comonomer) or random copolymers (with varying molar ratios of two different $\mathrm{A}_{2}$ comonomers). The latter approach gives valuable information on the optimal incorporation (mol. \%) of comonomers to ensure a complete bathochromic shift in fluorescence emission in the resultant CPNs while minimising detrimental effects on quantum yield that were found to arise from increased interchain aggregation at higher levels of incorporation. The compatibility of these nanoparticles with standard cellular imaging and analysis techniques, such as microscopy and flow cytometry, has been assessed.

\section{Experimental}

\subsection{Materials}

2,7-Dibromo-9,9-dioctyl-9H-fluorene, 1, was synthesised from 2,7-dibromofluorene (97\%, Sigma Aldrich) using standard literature procedures.[25, 26] 2,2'-(9,9-Dioctyl-9H-fluorene2,7-diyl)bis(4,4,5,5-tetramethyl-1,3,2-dioxaborolane), 2, was prepared from $\mathbf{1}$, again using a standard literature procedure.[27] 4,7-Dibromo-2,1,3-benzothiadiazole, 3, was prepared in two steps from o-phenylenediamine ( $>99 \%$, Sigma Aldrich) by modification of a literature method.[28] The method employed was identical save that in the first step direct extraction into dichloromethane of the intermediate, 2, 1, 3-benzothiadiazole, from acid solution was employed rather than steam distillation, and the isolated compound was then directly 
brominated to give $\mathbf{3}$. The spectroscopic data for each compound was in agreement with that reported in the literature. Amberlite XAD-2 resin, tetrakis(triphenylphosphine)palladium(0) and monomer $\mathbf{4}$ were purchased from Sigma Aldrich. All commercially sourced compounds were used as received, without further purification.

\subsection{Miniemulsion Polymerisation}

The following procedure for the synthesis of NP1a is representative of the method used to synthesise all of the CPN samples discussed. Sodium dodecyl sulfate (50.0 mg) and deionized water $(20 \mathrm{~mL})$ were transferred to a Schlenk tube and the resultant solution was degassed by bubbling with argon for 20 minutes. Monomers 1 (50.0 mg, $9.12 \times 10^{-2} \mathrm{mmol}$ ) and 2 (58.6 mg, $\left.9.12 \times 10^{-2} \mathrm{mmol}\right)$ were dissolved in toluene $(1.00 \mathrm{~mL})$, to which hexadecane (78 $\mu \mathrm{L})$ was also added, and this solution was degassed for 5 minutes in the same manner. Tetrakis(triphenylphosphine)palladium(0) (2.2 $\left.\mathrm{mg}, 1.90 \times 10^{-3} \mathrm{mmol}\right)$ was added to the monomer solution, which was then transferred to the reaction vessel. The reaction mixture was emulsified by ultrasonication (Cole Parmer 750W ultrasonicator, fitted with microtip, set to $22 \%$ power) for 2 minutes while cooling with an ice bath. The Schlenk tube was resealed and the miniemulsion was heated to $72{ }^{\circ} \mathrm{C}$, followed by addition of $1 \mathrm{M}$ aqueous sodium hydroxide solution (365 $\mu \mathrm{L}$ ), and the reaction mixture was stirred for 16 hours. After cooling to room temperature, the cap of the reaction vessel was removed and the emulsion was stirred for 5 hours to remove the residual toluene. The effective conjugated polymer concentration generated in this particular miniemulsion is approx. $3500 \mathrm{ppm}$, higher than that typical achievable via nanoprecipitation.

Note: For NP1b, the amount of water used in the reaction was halved (10 mL) compared to the amount used for synthesis of NP1a. This equates to an effective conjugated polymer concentration in the resultant miniemulsion of approx. 7000 ppm. For NP2a-c and NP3a-b 
the only change to the procedure described for NP1a is the choice of comonomers and the molar ratio of these monomers (Table 1). In each reaction, the total number of moles of the combined comonomers was $1.82 \times 10^{-1} \mathrm{mmol}$.

\subsection{Surfactant Removal}

To prepare the nanoparticle dispersions for use in cellular assays, a $4 \mathrm{~mL}$ aliquot of the asprepared dispersion was added to $200 \mathrm{mg}$ of Amberlite XAD-2 resin (prewashed twice with deionised water) and this suspension was shaken at room temperature for one hour. The resin was removed by decantation of the aqueous nanoparticle dispersion (using a syringe with a fine needle) and a fresh batch of resin (200 mg) was subsequently added. After shaking for a further hour, the nanoparticle dispersion was similarly decanted and then filtered through glass wool to remove any residual beads of the Amberlite XAD-2.

\subsection{Characterisation}

\subsubsection{DLS Analysis}

DLS particle size analysis of the nanoparticles in water was carried out after dilution of the as-prepared aqueous nanoparticle dispersion $(400 \mu \mathrm{L})$ with deionised water $(1.6 \mathrm{~mL})$ and surfactant removal (as described above, using 2 x 20 mg of Amberlite XAD-2), using a Malvern Zetasizer Nano ZS. All DLS particle size analyses were carried out at a controlled temperature of $20^{\circ} \mathrm{C}$.

\subsubsection{GPC Analysis}

In order to prepare THF solutions of the linear CPs that comprise NP1a-b, NP2a-c and NP3a-b, a $200 \mu \mathrm{L}$ aliquot of the as-prepared nanoparticle dispersion was flocculated through addition of $1.3 \mathrm{~mL}$ methanol and the polymer was isolated by centrifugation $(14,000 \mathrm{rpm}, 1$ minute) and decantation of the supernatant. The polymer was dried in air to remove residual 
methanol before dissolving in tetrahydrofuran $(1 \mathrm{~mL})$ and the resultant solution was filtered through a $0.45 \mu \mathrm{m}$ syringe filter. GPC analysis was carried out at $35{ }^{\circ} \mathrm{C}$ using a Viscotek GPCmax VE2001 solvent/sample module with $2 \times$ PL gel $10 \mu \mathrm{m}$ Mixed-B and PL gel 500A columns, a Viscotek VE3580 RI detector and a VE 3240 UV/VIS 50 multichannel detector. The flow rate was $1 \mathrm{~mL} / \mathrm{min}$ and the system was calibrated with low polydispersity polystyrene standards in the range of 200 to $180 \times 10^{4} \mathrm{~g} \mathrm{~mol}^{-1}$ from Agilent. The analysed samples contained n-dodecane as a flow marker.

\subsubsection{UV/Vis and PL Analysis}

For recording of absorption and emission spectra in water, the aqueous nanoparticle dispersion (as-prepared for DLS analysis, described above) was diluted with water to an optical density of between 0.5 and 1 . UV-Vis absorption spectra of the nanoparticles at this concentration were recorded on a Varian Cary 55 5000UV-Vis- NIR spectrophotometer at room temperature. PL spectra were recorded on a Varian Cary Eclipse fluorimeter.

\section{$<$ Table 1 inserted here $>$}

\subsubsection{PLQY Analysis}

All PLQY measurements were obtained using a Fluoromax-4 spectrofluorometer with integrating sphere attachment (instrument standardized with a Tungsten lamp). Nanoparticle suspensions in water were diluted to an optical density $<0.2$ for measurements. Background reference measurements were obtained using water $(600 \mu \mathrm{L})$ in 7 x $40 \mathrm{~mm}$ clear glass vials. Nanoparticle suspensions $(600 \mu \mathrm{L})$ were also measured in 7 x $40 \mathrm{~mm}$ clear glass vials. An excitation wavelength of $390 \mathrm{~nm}$ was used for all samples apart from F8BT nanoparticles NP2c, for which the excitation wavelength was $440 \mathrm{~nm}$. To calculate PLQYs, four measurements were obtained: i) background scatter (e. g. $380-400$ or $430-450 \mathrm{~nm}$ ); ii) 
background fluorescence (400-760 nm); iii) sample scatter (e. g 380-400 nm or 430-450 nm); and iv) sample fluorescence (400-760 nm). Appropriate optical filters were used to avoid oversaturation of the detector during collection of scatter measurements and these filters were corrected for in the PLQY calculation. For each sample of nanoparticles, PLQY measurements were repeated in triplicate, using a fresh suspension after each set of sample scatter and sample fluorescence measurements. The reported PLQY value is therefore an average of three measurements.

\subsubsection{TEM Analysis}

Solutions of graphene oxide were synthesised as reported previously.[29] Aqueous solutions of graphene oxide $(0.10 \mathrm{mg} / \mathrm{mL})$ were sonicated for $30 \mathrm{~s}$ prior to use. Lacey carbon grids (400 Mesh, Cu) (Agar Scientific) were cleaned using air plasma from a glow-discharge system $(2 \mathrm{~min}, 20 \mathrm{~mA})$. The TEM grids were placed on a filter paper and one drop $(\approx 0.08$ $\mathrm{mL}$ ) of the sonicated GO solution was deposited onto each grid from a height of $\approx 1 \mathrm{~cm}$, allowing the filter paper to absorb the excess solution, and the grids were left to air-dry in a desiccator cabinet for $\approx 60 \min .5 \mu \mathrm{L}$ of the nanoparticle dispersion $(100 \mathrm{ppm})$ was pipetted onto a GO grid and blotted away with filter paper after 2 minutes, the grid was then left to air-dry in a desiccator cabinet for $\approx 60 \mathrm{~min}$. Brightfield TEM images were captured with a transmission electron microscope (JEOL 2000FX), operating at $200 \mathrm{kV}$. Average sizes of the nanoparticles were determined from counting the sizes of 200 particles for each sample.

\subsubsection{Cell Culture}

HCT116, A549 and MCF-7 cells were obtained from ATCC. All cell lines were maintained in RPMI-1640 media supplemented with $10 \%$ heat inactivated fetal calf serum and $0.2 \mathrm{mM}$ L-glutamine in a humidified incubator with $5 \% \mathrm{CO}_{2}$. 


\subsubsection{Viability Assay}

Cells were seeded in 96 well plates at 10,000 cells per well 24 hours prior to use. Cells were incubated with particles diluted in Optimem media (Life Technologies) at the stated concentrations for 24 hours. The media was aspirated and the cells washed once in PBS. The cells were then incubated with Alamar Blue reagent (Life technologies) for 1 hour and fluorescence was measured at excitation $585 \mathrm{~nm}$ emission $610 \mathrm{~nm}$.

\subsubsection{Confocal Microscopy}

HCT-116 cells were seeded on cover glass slides. After 24 hours they were incubated with particles (50 $\mu \mathrm{g} / \mathrm{ml}$ in Optimem) for 2 hours after which time they were washed three times in cold PBS. NP2c samples were counterstained with CellMask ${ }^{\mathrm{TM}}$ Deep Red Plasma membrane stain (Life Technologies) and Hoechst 33342 for 5 minutes at $37^{\circ} \mathrm{C}$. Cells were fixed for 5 minutes at room temperature in $4 \%$ paraformaldehyde-PBS prior to imaging using a LSM710 META confocal microscope (Zeiss).

\subsubsection{Flow Cytometry}

Cells were seeded on 6 well tissue culture plates for 24 hours. They were incubated with 50 $\mu \mathrm{g} / \mathrm{ml}$ particles for 2 hours then washed three times in ice cold PBS. Cells were trypsinized and fixed in $4 \%$ paraformaldehyde -PBS for 5 minutes at room temperature. Cells were resuspended in PBS and analysed by flow cytometry within 24 hours using a MoFlo XDP flow cytometer (Beckman Coulter). Flow cytometer settings were NP1a: 405 nm excitation, emission 417.4-482.5 nm; NP2c: 488 nm excitation, emission 515-543 nm; NP3a: 488 nm excitation, 655-685 nm emission.

$<$ Fig. 1. inserted here $>$

<Fig. 2. inserted here $>$ 


\section{Results and Discussion}

\subsection{Miniemulsion Polymerisation and Surfactant Removal}

Initial efforts to develop a versatile route for the direct synthesis of CPNs by Suzuki reaction of $A_{2}+B_{2}$ monomers focused on the synthesis of CPNs of PFO via copolymerisation of equimolar amounts of 2,7-dibromo-9,9-dioctyl-9H-fluorene, 1, and 2,2'-(9,9-dioctyl-9Hfluorene-2,7-diyl)bis(4,4,5,5-tetramethyl-1,3,2- dioxaborolane), 2. The miniemulsion reactions remain stable at dispersed/continuous phase ratios of 1:20 and 1:10. These are higher than previously reported (1:25), which has advantages in terms of potential for scaleup.[23] Previous reports showed that varying the surfactant concentration from $1-0.08$ wt. \% decreased the mean particle diameter from 85 to $39 \mathrm{~nm}$.[23] In this contribution, which is primarily focused on varying the copolymer structure, a surfactant concentration close to the minimum amount necessary to provide a stable miniemulsion throughout the reaction was used, e.g. 0.25 and 0.5 wt. \% for dispersed/continuous phase ratios of 1:20 and 1:10, respectively. The use of a low surfactant concentration minimised the number of purification cycles necessary for removal of free surfactant in the final dispersions. TEM analysis of NP1a and NP1b (produced with dispersed/continuous phase ratios of 1:20 and 1:10 respectively), showed that nanoparticles obtained by this method generally have a spherical morphology (see Figure 1). There is some disparity in size between the distributions obtained via DLS and TEM for all samples (see Table 1), due to the relatively polydisperse nature of the samples. However, both methods are in agreement that the majority of particles present in all samples are below $100 \mathrm{~nm}$ in diameter and thus readily amendable to entry into cells by active mechanisms, e.g. endocytosis.[30] Moreover, CPNs prepared by precipitation with a similar level of polydispersity within this size regimen have been successfully employed in real-time sentinel lymph node mapping in vivo.[7] 
Purification of the CPNs for application in cellular imaging requires removal of the organic solvent (toluene) and free surfactant. Removing the lid of the reaction vessel and stirring at room temperature for 5 hours achieved the former, while the latter can be achieved by an exhaustive dialysis of the dispersion (7 days, changing the water twice daily). However, dialysis is both time-consuming, causing a considerable bottleneck in CPN production, and expensive, hence an alternative strategy was investigated. In protein purification detergent removal (ionic and non-ionic surfactants) can be achieved by the use of a porous, hydrophobic polystyrene resin, Amberlite XAD-2.[31, 32] It was found that for the CPNs prepared in this work it was possible to remove both the residual toluene and the SDS to a final level that is below the surfactant CMC by simply shaking the crude nanoparticle suspension (as a 5-fold dilution) with 1 wt. \% of Amberlite XAD-2 for 15 minutes and then repeating this process with a fresh batch of resin. Analysis of the samples purified by this method by DLS shows no detrimental effects on the CPN size or polydispersity (Table 1). Unlike dialysis, purification using the resin does not remove the aqueous base (sodium hydroxide) employed in the polymerisation, however it was at a sufficiently low level that the as-purified CPN suspension could be added directly to biological buffer solutions without the need for further purification. Using this approach it is possible to produce sub-100 nm PFO nanoparticles at high concentrations (NP1b, Table 1), however these more concentrated samples (1:10, dispersed/continuous) tended to flocculate after surfactant removal by treatment with Amberlite XAD-2 resin upon standing for several hours. Hence in all subsequent copolymerisation reactions a dispersed/continuous phase ratio of 1:20 was employed as these dispersions can be stored for several months following surfactant removal, without dilution.

Measurement of UV/vis and PL spectra of NP1a in dilute aqueous suspension, indicated that the PFO adopts a mixture of glassy phase (disordered) and $\beta$-phase (ordered) chain 
morphologies within the nanoparticles (Figure 2). The $\beta$-phase is an ordered form of PFO in which the backbones of neighbouring polymer chains are planarized with respect to each other.[33] This is consistent with the findings of a number of previous reports in which aqueous dispersions of PFO nanoparticles have been processed from (or annealed with) a “good” solvent.[34, 35] The photoluminescence quantum yield (PLQY) of NP1a in water (38 \%) is similar to values reported for PFO NPs prepared by other methods.[23, 34] In order to assess the degree of polymerisation obtained by Suzuki polymerisation in a miniemulsion, small aliquots of the as-made CPN suspensions were flocculated through addition of methanol and the molecular weight of the isolated polymers (in THF) was determined by gel permeation chromatography (Table 1). The molecular weights of PFO recovered from NP1a and NP1b emulsions $\left(\mathrm{M}_{\mathrm{n}}=1.4-1.5 \times 10^{4} \mathrm{~g} \mathrm{~mol}^{-1}\right)$ compared favourably with those produced by reaction of an A-B monomer in the earlier report $\left(9.0 \times 10^{3}-2.3 \times 10^{4} \mathrm{~g} \mathrm{~mol}^{-}\right.$ ${ }^{1}$ ),[23] indicating that $\mathrm{A}_{2}+\mathrm{B}_{2}$ reactions can provide an acceptably high degree of polymerisation while providing the possibility of vastly expanding the structural diversity.

\subsection{Green-Emitting CPNs}

The emission wavelength of fluorene polymers can be tuned through copolymerisation of fluorene with various electron withdrawing co-monomers.[36, 37] Miniemulsion copolymerisation of dioctyl fluorene monomers $\mathbf{1}$ and $\mathbf{2}$ with benzothiadiazole monomer $\mathbf{3}$ gave CPN dispersions, NP2a-c, that luminesce primarily in the green (Table 2). NP2a and NP2b are comprised of random copolymers, synthesised by reaction of $\mathbf{1}$ and $\mathbf{2}$ with 5 and 10 mol. \% of 3, respectively. The UV/vis absorption spectra of NP2 are similar to those of NP1 (Figure 3) due to the relative abundance of fluorene-fluorene segments in these materials. The secondary absorption peak observed at $437 \mathrm{~nm}$ for NP2 shows that the polymers in these nanoparticles retain the ability to form the more ordered $\beta$-phase conformation, despite the 
incorporation of small amounts of the benzothiadiazole units.[38] The absorbance of the fluorene-benzothiadiazole segments in NP2 is observed as a broad shoulder that partially overlaps with the $\beta$-phase absorption and that extends to around $500 \mathrm{~nm}$ (Figure 3a). The PL emission of these copolymers (exciting at $390 \mathrm{~nm}$ ) is almost exclusively derived from the relatively few fluorene-benzothiadiazole chain segments, as a result of efficient Förster Resonance Energy Transfer (FRET) between these and the fluorene-fluorene segments (Figure 3b). As a result, the effective Stokes shift for both samples is large (154 nm). Increasing the amount of benzothiadiazole monomer 3 from 5 to $10 \mathrm{~mol}$. \% resulted in a significant increase in the PLQY (Table 2) of the resultant nanoparticles, NP2a and NP2b, with the value for the latter (56 \%) being much higher than those typically reported for green emitting CPNs.

<Fig. 3. inserted here $>$

<Fig. 4. inserted here >

This is analogous to the recent findings of McNeill et al., who report that CPNs comprised of blends of PFBT and MEH-PPV have a higher PLQY than CPNs comprised of PFBT alone.[39] Considering the relative complexity of the three monomer reaction, the observed polymer molecular weights (1.3-1.4 x $10^{4} \mathrm{~g} \mathrm{~mol}^{-1}$, Table 1) are encouraging, highlighting the robustness and efficiency of the miniemulsion polymerisation reactions. Polymerisation of a 1:1 molar ratio of $\mathbf{2}$ and $\mathbf{3}$ gave CPN dispersion NP2c, consisting of the well-studied alternating co-polymer F8BT.[36] The broad and featureless UV/vis and PL spectra (Figure 4) and moderate quantum efficiency (12\%) for NP2c are consistent with previous examples of F8BT CPNs that have been prepared by nanoprecipitation.[14] Incorporation of relatively small amounts (5-10\%) of benzothiadiazole into a random co-polymer based on PFO gives 
green-emitting CPNs with significantly higher quantum efficiencies than CPNs of the exactly alternating co-polymer (F8BT).

\section{<Table 2 inserted here $>$}

\section{<Fig. 5. Inserted here $>$}

\subsection{FR-NIR Emitting CPNs}

In order to facilitate their use for in vivo imaging applications, there is currently considerable focus on the development of CPNs that emit in the FR/NIR region.[5-9] However, this remains a considerable challenge due to the limited solubility of low bandgap conjugated polymers and their tendency to aggregate in the solid state. The former characteristic renders these polymers less suitable for post-polymerisation fabrication into CPNs via nanoprecipitation and the latter significantly impacts upon the PLQY of the resultant nanomaterials.[9] Incorporation of the thiophene-benzothiadiazole-thiophene monomer 4 (1 - 35 mol. \%) into PFO-based random copolymers in a conventional solution-based Suzuki coupling polymerisation has been shown to produce materials that display FR/NIR solid state emission when deposited as thin films.[37] Suzuki miniemulsion polymerisation reactions were carried out using $\mathbf{1}$ and $\mathbf{2}$ as comonomers, with 5 and $10 \mathrm{~mol}$. \% of $\mathbf{4}$ to give nanoparticle dispersions NP3a and NP3b, respectively. It was not possible to incorporate higher loadings of $\mathbf{4}$ into these dispersions due to the limited solubility of $\mathbf{4}$ in toluene at room temperature (the temperature at which the emulsification step is performed). In the case of NP3b, it was observed that a small amount of monomer 4 had precipitated during emulsification, and hence the maximum possible mol. \% for incorporation of this comonomer into CPNs by this method lies between 5 and $10 \mathrm{~mol}$. \%. The precipitation of monomer 4 resulted in a reduction in the molecular weight of the polymer $\left(5.7 \times 10^{3} \mathrm{~g} \mathrm{~mol}^{-1}\right)$ contained within these nanoparticles, when compared with those produced in the other miniemulsion 
reactions (see Table 1). GPC analysis of the polymer from NP3a indicated a number average molecular weight of $1.2 \times 10^{4} \mathrm{~g} \mathrm{~mol}^{-1}$, corresponding to a degree of polymerisation of around 30 and therefore an average of 3 segments derived from monomer 4 per chain. UV/vis absorption spectra of NP3a and NP3b indicated that the more abundant fluorene-fluorene segments of the polymer chains can adopt glassy phase and $\beta$-phase conformations (Figure 5a). The $\beta$-phase absorption is significantly lower for composition NP3b than NP3a. The absorption spectra also have a broad absorption peak with a maximum at $529 \mathrm{~nm}$, associated with the less abundant, lower bandgap segments derived from monomer $\mathbf{4}$. The emission spectra of NP3a and NP3b exhibited primarily red fluorescence $\left(\lambda_{\mathrm{em}}=630 \mathrm{~nm}\right.$, exciting at $390 \mathrm{~nm}$ ), with a significant amount of this fluorescence encompassing the FR/NIR region (Figure 5b). Unlike the green-emitting CPNs discussed above, in which complete energy transfer from the blue to green segments is observed in all cases, NP3a and NP3b both exhibit a small amount of fluorescence in the blue region, corresponding to emission from segments of $\beta$-phase PFO. Previous attempts to form efficient FR/NIR CPNs have focused on the nanoprecipitation of poly[(9,9-dihexylfluorenyl-co-2,1,3-benzothiadiazole)-co-4,7di(thiophen-2-yl)-2,1,3-benzothiadiazole (PFBTDBT), in which the lower energy fluorenebenzothiadiazole (green-emitting) segments have a better overlap with the higher energy, redsegments than the fluorene-fluorene segments (blue-emitting) segments found in NP3.[5] Despite evidence of some blue emission, NP3a has a high PLQY (34 \%) by comparison with alternative FR/NIR nanoparticles and are thus promising candidates for in vivo bioimaging applications. NP3b, with 10 mol. \% of monomer 4, has a slightly lower PLQY (19 \%). Factors that may contribute to the lower efficiency of NP3b when compared to NP3a are the lower molecular weight of the CP chains in NP3b and the increased interchain aggregation caused by the higher mol. \% of red-emitting segments. This is evidenced by the reduced amount of PFO $\beta$-phase observed in the UV/vis spectrum of NP3b (Figure 5b). 
<Fig. 6. inserted here $>$

<Fig. 7. inserted here $>$

<Fig. 8. inserted here $>$

\subsection{Cellular Imaging and Cytotoxicity Assays}

The suitability of nanoparticle dipersions NP1a, NP2c and NP3a (blue, green and red emitting, respectively) for bioimaging was tested in 2D cell cultures. Each type of nanoparticle was shown to be detectable by flow cytometry at $50 \mu \mathrm{g} / \mathrm{mL}$ after a 2 hour incubation, demonstrating the potential to utilize these materials for FACS analysis experiments (Figure 6). Confocal microscopy revealed rapid internalisation of the CPNs into HCT116 cells in all cases, with a largely perinuclear localisation (Figure 7). However NP3a showed significant bleed though into blue, green and red channels making them impractical for multiplexing with many standard imaging reagents such as Hoechst 33342 and DAPI. In contrast, NP2c was compatible with reagents including Hoechst 33342, DAPI, CellMask ${ }^{\mathrm{TM}}$ Deep Red Plasma membrane (Figure 7) and red fluorescent protein (RFP) (data not shown) demonstrating that it is a good candidate for use in microscopy studies. The cytotoxicity of nanoparticles NP1a, NP2c and NP3a was tested using the Alamar Blue assay. After incubation for 24 hours the particles reduced viability by a maximum of $25 \%$ of untreated controls (Table 3). At higher concentrations the viability dropped further (Figure 8) however such high concentrations were well beyond those necessary for bioimaging purposes.

\section{<Table 3. inserted here $>$}

\section{Conclusions}

We have demonstrated the wider utility of Suzuki emulsion polymerisation for the direct synthesis of CPNs with tunable fluorescence emission. Their useful optical properties, such 
as high PLQYs, and small size $(<100 \mathrm{~nm})$ render these nanoparticles suitable for use in biological applications, such as cellular imaging and sorting via fluorescence microscopy and flow cytometry. The methodology developed is straightforward in terms of both synthesis and purification and more readily scalable than many previously reported methods for producing CPNs. Through developing a deeper understanding of the mechanism of Suzuki emulsion polymerisation reactions and, in particular, the factors that control particle size, and use of biocompatible surfactants, one pot synthesis of CPNs has the potential to supersede current indirect methodologies (e. g. nanoprecipitation) and to support the wider use of these materials in biomedical imaging.

\section{References}

[1] L.P. Fernando, P.K. Kandel, J. Yu, J. McNeill, P.C. Ackroyd, K.A. Christensen, Biomacromolecules, $11(2010)$ 2675-2682.

[2] J. Lee, M. Twomey, C. Machado, G. Gomez, M. Doshi, A.J. Gesquiere, J.H. Moon, Macromolecular Bioscience, 13 (2013) 913-920.

[3] M. Green, P. Howes, C. Berry, O. Argyros, M. Thanou, Proceedings of the Royal Society A: Mathematical, Physical and Engineering Sciences, 465 (2009) 2751-2759.

[4] R. Ahmad Khanbeigi, T.F. Abelha, A. Woods, O. Rastoin, R.D. Harvey, M.-C. Jones, B. Forbes, M.A. Green, H. Collins, L.A. Dailey, Biomacromolecules, 16 (2015) 733-742.

[5] D. Ding, J. Liu, G. Feng, K. Li, Y. Hu, B. Liu, Small, 9 (2013) 3093-3102.

[6] K. Li, D. Ding, D. Huo, K.-Y. Pu, N.N.P. Thao, Y. Hu, Z. Li, B. Liu, Advanced Functional Materials, 22 (2012) 3107-3115, S3107/3101-S3107/3107.

[7] S. Kim, C.-K. Lim, J. Na, Y.-D. Lee, K. Kim, K. Choi, J.F. Leary, I.C. Kwon, Chemical Communications (Cambridge, United Kingdom), 46 (2010) 1617-1619.

[8] C. Wu, S.J. Hansen, Q. Hou, J. Yu, M. Zeigler, Y. Jin, D.R. Burnham, J.D. McNeill, J.M. Olson, D.T. Chiu, Angewandte Chemie, International Edition, 50 (2011) 3430-3434, S3430/3431-\$3430/3414. 
[9] J. Liu, G. Feng, D. Ding, B. Liu, Polymer Chemistry, 4 (2013) 4326-4334.

[10] A.L. Koner, D. Krndija, Q. Hou, D.J. Sherratt, M. Howarth, ACS Nano, 7 (2013) 1137-1144.

[11] M.C. Mancini, B.A. Kairdolf, A.M. Smith, S. Nie, Journal of the American Chemical Society, 130 (2008) 10836-10837.

[12] C. Szymanski, C. Wu, J. Hooper, M.A. Salazar, A. Perdomo, A. Dukes, J. McNeill, Journal of Physical Chemistry B, 109 (2005) 8543-8546.

[13] C. Wu, C. Szymanski, J. McNeill, Langmuir, 22 (2006) 2956-2960.

[14] C. Wu, B. Bull, C. Szymanski, K. Christensen, J. McNeill, ACS Nano, 2 (2008) 2415-2423.

[15] K. Landfester, R. Montenegro, U. Scherf, R. Guntner, U. Asawapirom, S. Patil, D. Neher, T. Kietzke, Advanced Materials (Weinheim, Germany), 14 (2002) 651-655.

[16] K. Li, R. Zhan, S.-S. Feng, B. Liu, Analytical Chemistry (Washington, DC, United States), 83 (2011) $2125-2132$.

[17] T. Kietzke, D. Neher, K. Landfester, R. Montenegro, R. Guentner, U. Scherf, Nature Materials, 2 (2003) 408-412.

[18] E. Hittinger, A. Kokil, C. Weder, Angewandte Chemie, International Edition, 43 (2004) 18081811.

[19] J. Pecher, J. Huber, M. Winterhalder, A. Zumbusch, S. Mecking, Biomacromolecules, 11 (2010) 2776-2780.

[20] M.C. Baier, J. Huber, S. Mecking, Journal of the American Chemical Society, 131 (2009) 1426714273.

[21] R. Wang, C. Zhang, W. Wang, T. Liu, Journal of Polymer Science, Part A: Polymer Chemistry, 48 (2010) 4867-4874.

[22] H. Li, X. Wu, B. Xu, H. Tong, L. Wang, RSC Advances, 3 (2013) 8645-8648.

[23] C. Negele, J. Haase, A. Leitenstorfer, S. Mecking, ACS Macro Letters, 1 (2012) 1343-1346.

[24] D. Muenmart, A.B. Foster, A. Harvey, M.-T. Chen, O. Navarro, V. Promarak, M.C. McCairn, J.M. Behrendt, M.L. Turner, Macromolecules (Washington, DC, United States), 47 (2014) 6531-6539. 
[25] R. Grisorio, P. Mastrorilli, C.F. Nobile, G. Romanazzi, G.P. Suranna, D. Acierno, E. Amendola, Macromolecular Chemistry and Physics, 206 (2005) 448-455.

[26] M.R. Craig, M.M. de Kok, J.W. Hofstraat, A.P.H.J. Schenning, E.W. Meijer, Journal of Materials Chemistry, 13 (2003) 2861-2862.

[27] S.Y. Cho, A.C. Grimsdale, D.J. Jones, S.E. Watkins, A.B. Holmes, Journal of the American Chemical Society, 129 (2007) 11910-11911.

[28] Y. Jin, Y. Kim, S.H. Kim, S. Song, H.Y. Woo, K. Lee, H. Suh, Macromolecules (Washington, DC, United States), 41 (2008) 5548-5554.

[29] N.R. Wilson, P.A. Pandey, R. Beanland, R.J. Young, I.A. Kinloch, L. Gong, Z. Liu, K. Suenaga, J.P. Rourke, S.J. York, J. Sloan, ACS Nano, 3 (2009) 2547-2556.

[30] J. Rejman, V. Oberle, I.S. Zuhorn, D. Hoekstra, Biochemical Journal, 377 (2004) 159-169.

[31] P.S.J. Cheetham, Analytical Biochemistry, 92 (1979) 447-452.

[32] M. Hiraide, T. Shima, H. Kawaguchi, Analytical Sciences, 10 (1994) 505-507.

[33] A. Monkman, C. Rothe, S. King, F. Dias, Advances in Polymer Science, 212 (2008) 187-225.

[34] C. Wu, J. McNeill, Langmuir, 24 (2008) 5855-5861.

[35] J.M. Behrendt, A.B. Foster, M.C. McCairn, H. Willcock, R.K. O'Reilly, M.L. Turner, Journal of Materials Chemistry C: Materials for Optical and Electronic Devices, 1 (2013) 3297-3304.

[36] Y. He, S. Gong, R. Hattori, J. Kanicki, Applied Physics Letters, 74 (1999) 2265-2267.

[37] Q. Hou, Y. Xu, W. Yang, M. Yuan, J. Peng, Y. Cao, Journal of Materials Chemistry, 12 (2002) 28872892.

[38] D.W. Bright, F.B. Dias, F. Galbrecht, U. Scherf, A.P. Monkman, Advanced Functional Materials, 19 (2009) 67-73.

[39] X. Wang, L.C. Groff, J.D. McNeill, Journal of Physical Chemistry C, 118 (2014) 25731-25739. 


\section{Figure Captions}

Scheme 1. Selection of monomers employed in miniemulsion polymerisation reactions and resultant copolymers from which CPNs are comprised.

Table 1. Composition, particle size and copolymer molecular weight of CPNs synthesized via Suzuki polymerisation in a miniemulsion.

Fig. 1. TEM of PFO nanoparticles prepared with dispersed/continuous phase ratios of 1:20, NP1a, and 1:10, NP1b (scale bar $=100 \mathrm{~nm}$ ).

Fig. 2. UV/vis and PL spectra $\left(\lambda_{\text {ex }}=390 \mathrm{~nm}\right)$ of NP1a in water.

Fig. 3. a) UV/vis and b) PL emission spectra $\left(\lambda_{\mathrm{ex}}=390 \mathrm{~nm}\right)$ of NP2a and NP2b in water.

Fig. 4. UV/vis and PL emission spectra $\left(\lambda_{\mathrm{ex}}=465 \mathrm{~nm}\right)$ of NP2c in water.

Table 2. Optical properties of CPNs in water.

Fig. 5. a) UV/vis and b) PL emission spectra $\left(\lambda_{\text {ex }}=390 \mathrm{~nm}\right)$ of NP3a and NP3b in water.

Fig. 6. Flow cytometric analysis: HCT116 cells incubated with $50 \mathrm{ug} / \mathrm{ml}$ of nanoparticles NP1a, NP2c or NP3a for 2 hours. Cell only: filled histogram; nanoparticle incubated cells: unfilled histogram. Calculated \% of nanoparticle-labelled cells shown in each case.

Fig. 7. Confocal microscopy of particles in HCT116 cells. After a 2 hour incubation cells were either fixed (NP1a, NP3a) or stained with Hoechst 33342 and CellMask ${ }^{\mathrm{TM}}$ Deep Red Plasma membrane prior to fixing (NP2c) and visualisation by confocal microscopy (Scale bar = $10 \mu \mathrm{m})$. NP1a: particles-blue; NP2c: nucleus-blue, particles-green, plasma membrane-red; NP3a: particles-red. 
Fig. 8. Cytotoxicity of particles in three cell lines. Cells HCT116, MCF7, A549 were incubated with particles at the stated concentrations for 24 hours after which time the cell viability was measured using Alamar blue assay. $\mathrm{N}=4$, error bar SD.

Table 3. Mean cell viability after 24 hour incubation with $50 \mu \mathrm{g} / \mathrm{ml}$ of particles. 


\section{Schemes and Figures}

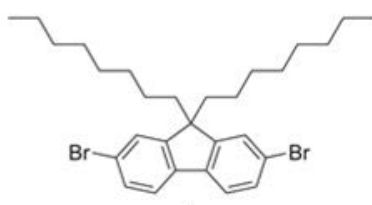

1
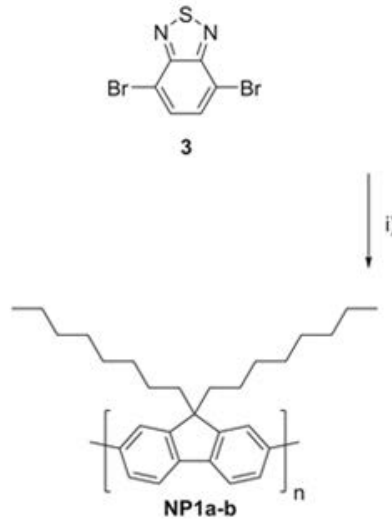

$\longrightarrow$

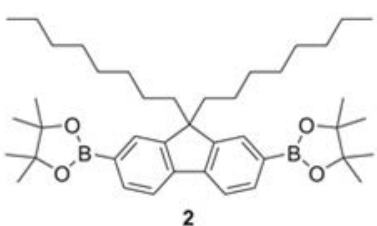

2

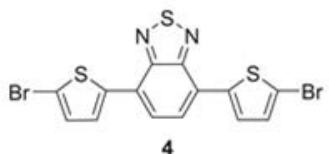

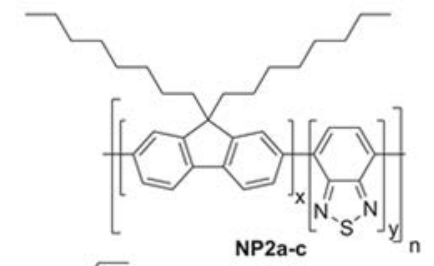

i) toluene, $\mathrm{n}$-hexadecane, $\mathrm{NaOH}, \mathrm{Pd}\left(\mathrm{PPh}_{3}\right)_{4}$, sodium dodecylsulfate, water, $72^{\circ} \mathrm{C}, 16 \mathrm{hr}$

\section{Scheme 1}

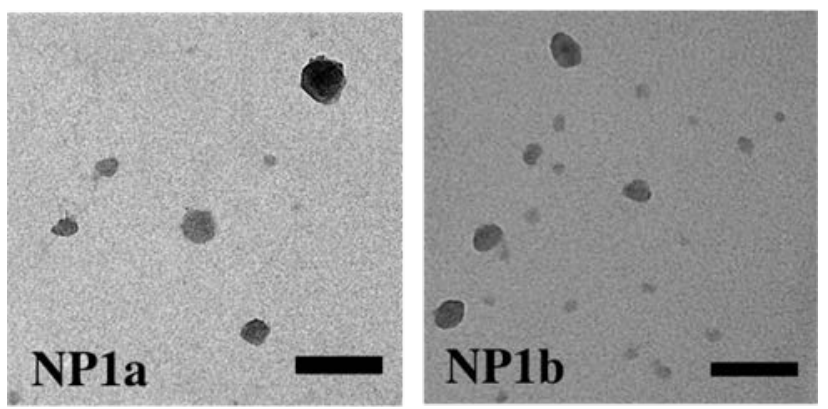

Fig. 1. 


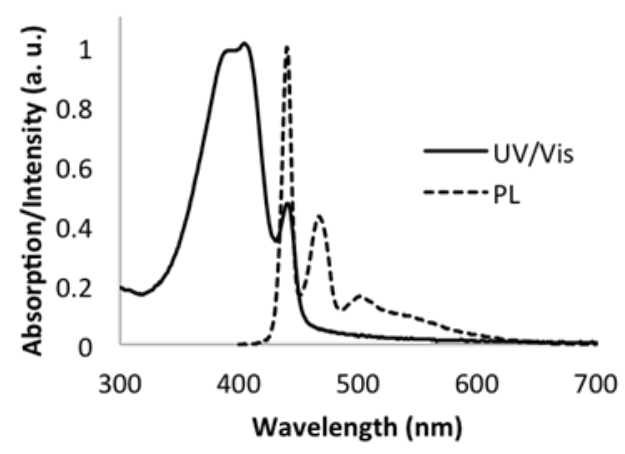

Fig. 2.
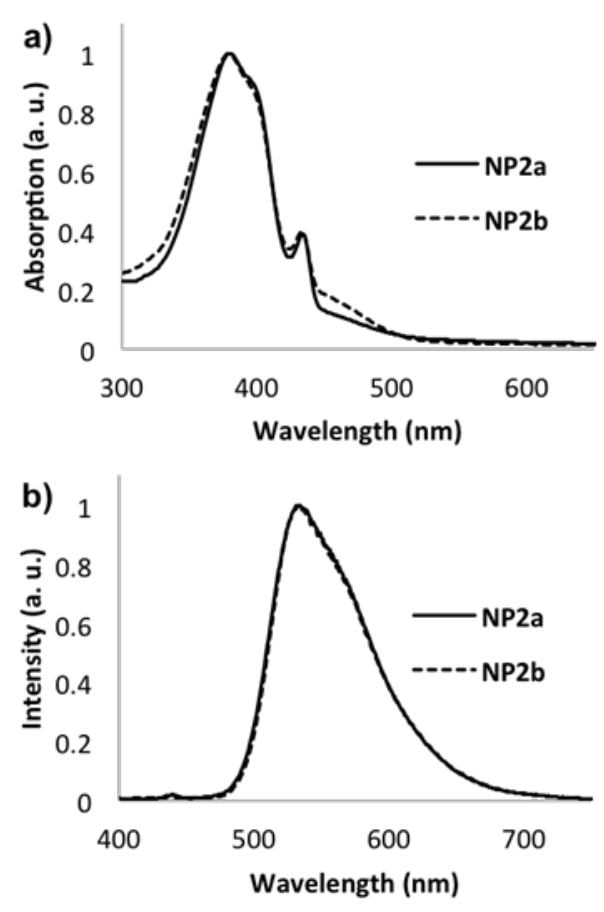

Fig. 3.

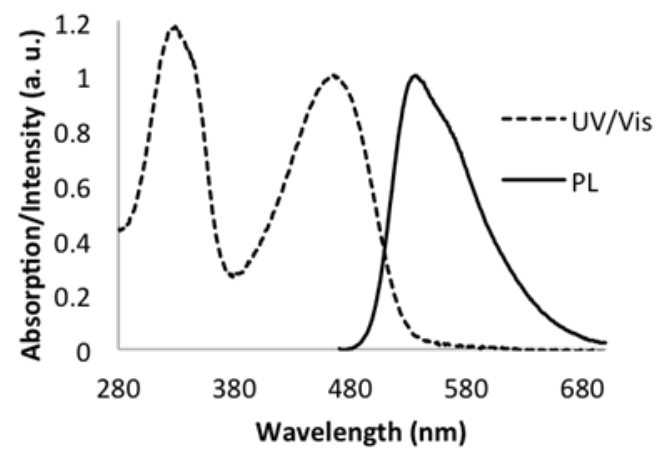

Fig. 4. 

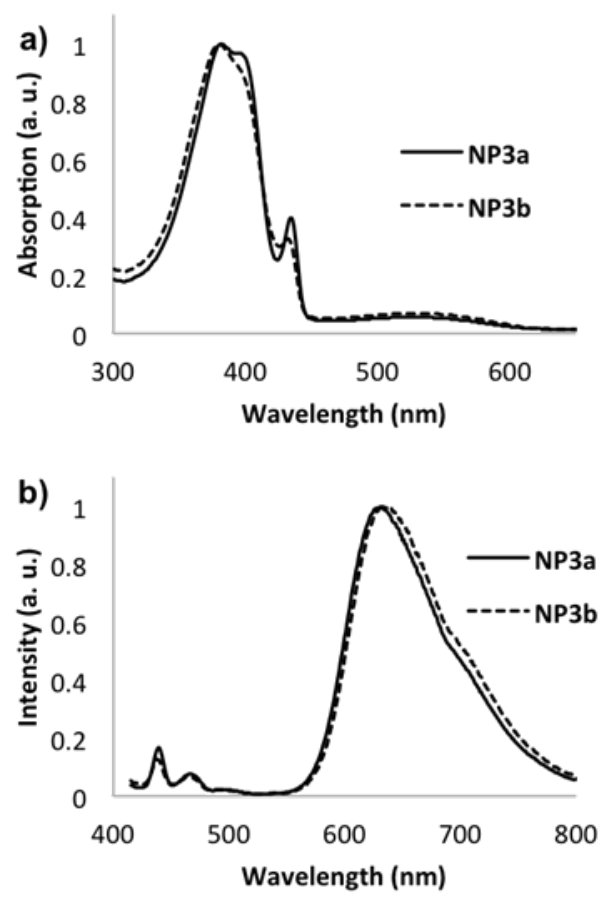

Fig. 5.
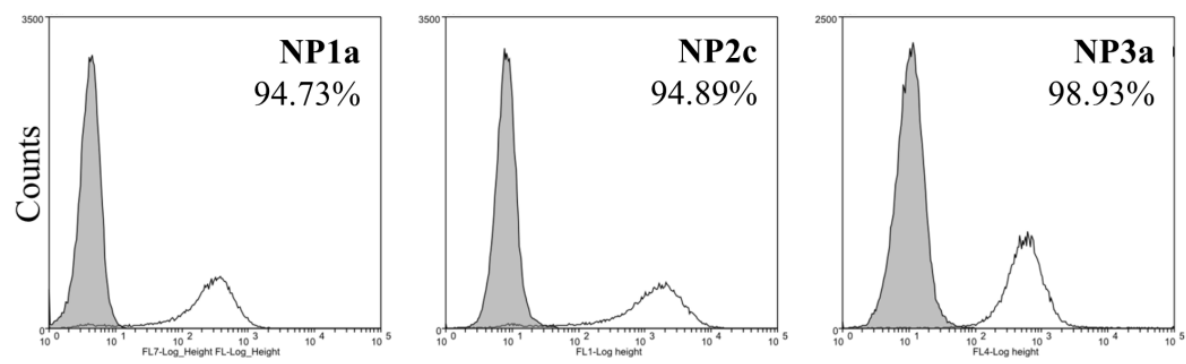

Fluorescence Intensity $\left(\log _{10}\right)$

Fig. 6. 

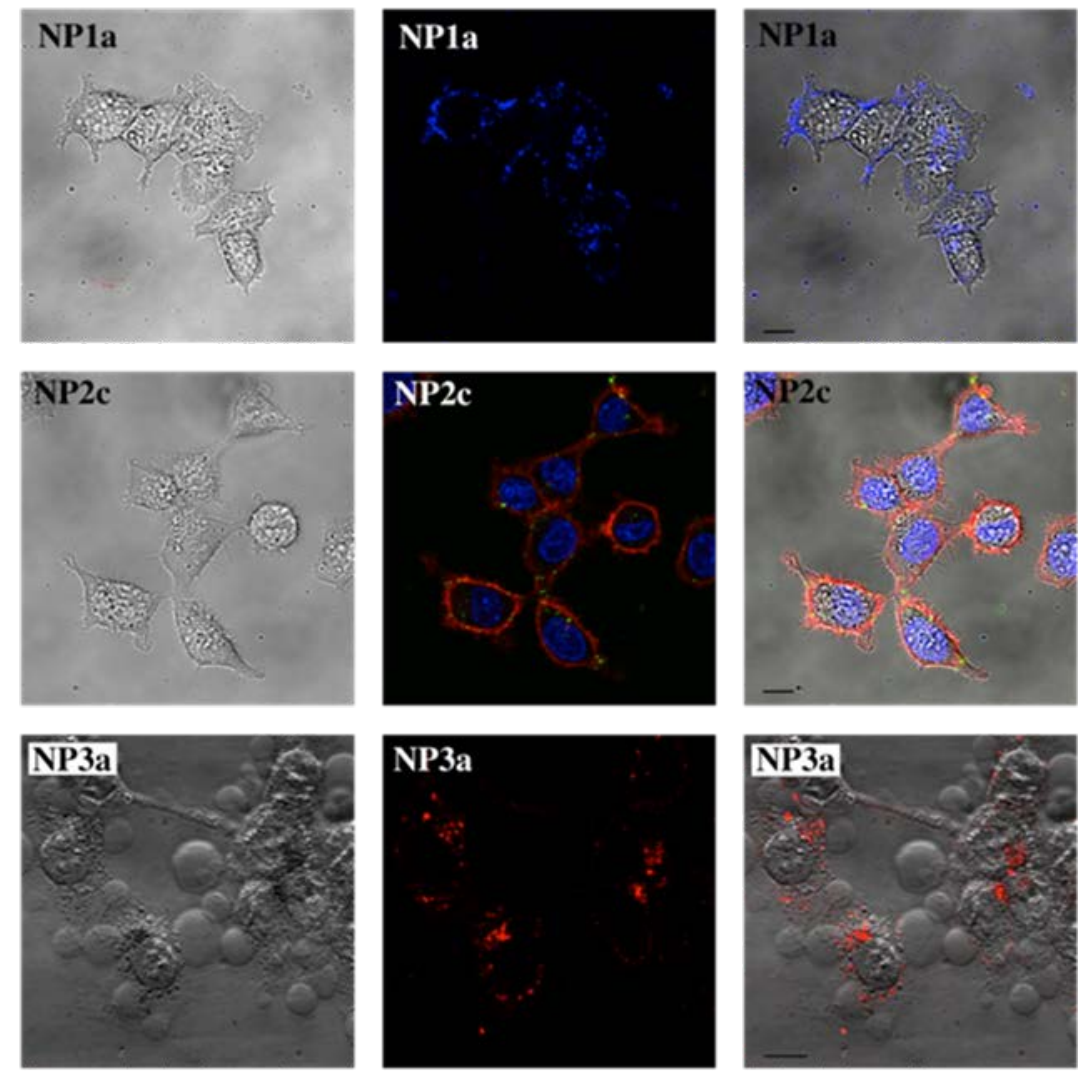

Fig. 7.
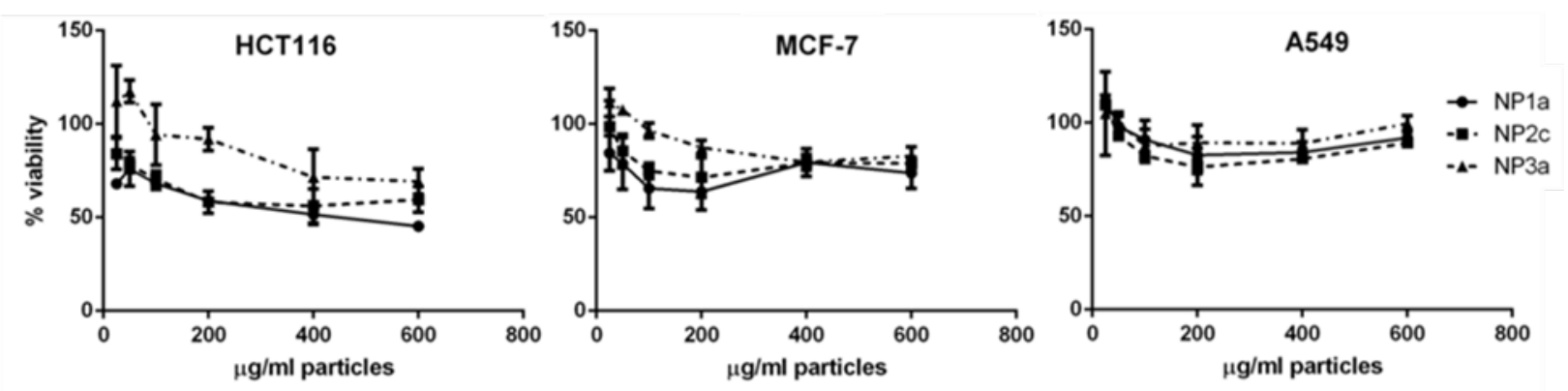

Fig. 8. 


\section{Tables}

\section{Table 1}

\begin{tabular}{llllllll} 
Sample code & Co-monomers & $\begin{array}{l}\text { Co-monomer } \\
\text { ratio (mol. } \%)\end{array}$ & $\begin{array}{l}\mathrm{D}_{\mathrm{DLS}}{ }^{\mathrm{a}} \\
(\mathrm{nm})\end{array}$ & $\mathrm{PdI}^{\mathrm{b}}$ & $\begin{array}{l}\mathrm{D}_{\mathrm{TEM}}{ }^{\mathrm{c}} \\
(\mathrm{nm})\end{array}$ & $\begin{array}{l}\mathrm{M}_{\mathrm{n}}{ }^{\mathrm{d}} \\
\left(\mathrm{g} \mathrm{mol}^{-1}\right)\end{array}$ & $\mathrm{M}_{\mathrm{w}} / \mathrm{M}_{\mathrm{n}}{ }^{\mathrm{d}}$ \\
\hline NP1a & $1+2$ & $50: 50$ & 71 & 0.16 & $23 \pm 12$ & $1.4 \times 10^{4}$ & 2.4 \\
NP1b & $1+2$ & $50: 50$ & $43(35 \%)$, & 0.20 & $19 \pm 9$ & $1.5 \times 10^{4}$ & 2.4 \\
& & & $13(65 \%)^{\mathrm{f}}$ & & & & \\
NP2a & $1+2+3$ & $45: 50: 5$ & 45 & 0.17 & $24 \pm 15$ & $1.3 \times 10^{4}$ & 2.9 \\
NP2b & $1+2+3$ & $40: 50: 10$ & 28 & 0.16 & $21 \pm 8$ & $1.4 \times 10^{4}$ & 2.8 \\
NP2c & $2+3$ & $50: 50$ & 73 & 0.16 & $23 \pm 15$ & $9.4 \times 10^{3}$ & 2.3 \\
NP3a & $1+2+4$ & $45: 50: 5$ & 55 & 0.19 & $25 \pm 11$ & $1.2 \times 10^{4}$ & 1.9 \\
NP3b & $1+2+4$ & $40: 50: 10$ & $59(75 \%)$, & 0.17 & $21 \pm 11$ & $5.7 \times 10^{3}$ & 3.4
\end{tabular}

a Mean diameter by number; b polydispersity index for the Gaussian size distribution measured via DLS; c mean diameter determined measuring 200 particles from TEM images; d molecular weight of polymer chains that comprise the nanoparticles following flocculation, centrifugation and dissolution in THF; e this sample was prepared with a dispersed/continuous phase ratio of 1:10, while this ratio was 1:20 for all other samples; $f$ biomodal size distribution indicated for this sample.

\section{Table 2}

\begin{tabular}{llll} 
Sample code & $\lambda_{\max }(\mathrm{nm})$ & $\lambda_{\mathrm{em}}(\mathrm{nm})$ & $\phi(\%)$ \\
\hline NP1a & 405 & 440 & $38 \pm 2$ \\
NP1b & 405 & 440 & $23 \pm 2$ \\
NP2a & 380 & 534 & $41 \pm 3$ \\
NP2b & 379 & 534 & $56 \pm 1$ \\
NP2c & 334,466 & 537 & $12 \pm 1$ \\
NP3a & 382 & 631 & $34 \pm 3$ \\
NP3b & 380 & 633 & $19 \pm 1$
\end{tabular}


Table 3

\begin{tabular}{llll} 
Cells & NP1a & NP2c & NP3a \\
\hline A549 & $98 \%$ & $93 \%$ & $100 \%$ \\
HCT116 & $76 \%$ & $78 \%$ & $117 \%$ \\
MCF7 & $79 \%$ & $86 \%$ & $108 \%$
\end{tabular}

